\title{
Low concentrations of bilirubin inhibit activation of hepatic stellate cells in vitro
}

\author{
YINHE TANG, QIYU ZHANG, YEFAN ZHU, GANG CHEN and FUXIANG YU \\ Department of Hepatobiliary and Pancreatic Surgery, The First Affiliated Hospital, \\ Wenzhou Medical University, Wenzhou, Zhejiang 325000, P.R. China
}

Received November 19, 2015; Accepted December 2, 2016

DOI: $10.3892 / \mathrm{mmr} .2017 .6201$

\begin{abstract}
Hepatic stellate cell (HSC) activation serves a key role in liver fibrosis, and is associated with chronic liver diseases. Bilirubin, a product of heme degradation, has been demonstrated to have antioxidant properties. The present study investigated the effects of physiological concentrations of bilirubin on rat HSC activation. Rat HSCs were isolated and cultured for several generations to induce activation. The activated HSCs were subsequently treated with $0,1,10$ or $20 \mathrm{mg} / 1$ bilirubin and assayed for parameters of cell activation. As the bilirubin concentration increased, HSCs demonstrated reduced production of reactive oxygen species, reduced protein expression levels of $\alpha$-smooth muscle actin, a decreased mRNA expression ratio of tissue inhibitor of matrix metalloproteinase-1/matrix metalloproteinase-2, decreased proliferation and increased apoptosis. In conclusion, elevated bilirubin levels, within its physiological concentration range, appeared to inhibit HSC activation. These findings suggested a potential role for bilirubin in the treatment of fibrosis that requires further investigation.
\end{abstract}

\section{Introduction}

In their resting state, hepatic stellate cells (HSCs) are a group of quiescent pericytes that comprise 5-8\% of liver cells (1).

Correspondence to: Dr Fuxiang Yu or Dr Gang Chen, Department of Hepatobiliary and Pancreatic Surgery, The First Affiliated Hospital, Wenzhou Medical University, 157 Wenrui Avenue, Ouhai, Wenzhou, Zhejiang 325000, P.R. China

E-mail: yfxbmu@163.com

E-mail: cg_2188@126.com

Abbreviations: HSC, hepatic stellate cell; ROS, reactive oxygen species; SMA, smooth muscle actin; ECM, extracellular matrix; RBCs, red blood cells; FBS, fetal bovine serum; DMEM, Dulbecco's modified Eagle's medium; DCFH-DA, 2',7'-dichlorofluorescin diacetate; FITC, fluorescein isothiocyanate; CCK-8, Cell Counting Kit-8; RT-PCR, reverse transcription-polymerase chain reaction; MMP-2, matrix metalloproteinase-2; TIMP-1, tissue inhibitor of matrix metalloproteinase-1; HO, heme oxygenase

Key words: bilirubin, hepatic stellate cells, activation, proliferation, apoptosis
They contain a number of lipid droplets in the cytoplasm primarily involved in vitamin A storage (2). Upon liver injury, HSCs become activated, which converts these quiescent cells into proliferative, fibrogenic and contractile myofibroblast-like cells (3). Activated HSCs are the principal collagen-producing cells and are responsible for fibrosis in chronic liver disease. Aside from their key roles in fibrosis and extracellular matrix (ECM) remodeling, activated HSCs have additionally been implicated in the progression of hepatitis and liver carcinogenesis (4). Due to their critical function in liver pathogenesis, the majority of proposed antifibrotic therapeutic approaches focus on modulating HSC activation.

Bilirubin is a bile pigment resulting from the degradation of heme, which is one of the breakdown products of red blood cells (RBCs). It is produced by the liver and is present in small quantities in the blood; however, excessive quantities cause toxicity, primarily due to neonatal jaundice and the potential for mediating irreversible brain damage at high concentrations (5). The normal range of total bilirubin concentration in the human body is $2-12 \mathrm{mg} / \mathrm{l}$; elevated levels are observed in disorders involving liver cell damage, biliary disease and RBC-processing disorders (6). In addition, a physiological role for bilirubin as an antioxidant has been reported. Stocker et al (7) demonstrated that at physiological concentrations, bilirubin efficiently protected against oxidation of lipid membranes. The scavenging and antioxidant activities of bilirubin were revealed to be more potent than those of vitamins $\mathrm{C}$ and $\mathrm{E}$ against lipid peroxidation $(8,9)$. As neonates have impaired antioxidant defenses and are susceptible to oxygen free radical-mediated diseases, it is hypothesized that elevated bilirubin levels act as a defense mechanism to counteract increased exposure to reactive oxygen species (ROS) $(10,11)$.

ROS serve important roles in HSC activation and hepatic fibrosis. Increased ROS production and the resulting oxidative stress are commonly observed in alcoholic liver disease, hepatitis $\mathrm{C}$ virus infection and experimental models of liver fibrogenesis (12). Antioxidant agents have been demonstrated to mitigate HSC activation and hepatic fibrosis in various research models $(13,14)$. However, the effect of bilirubin on HSC activation remains to be investigated.

In the present study, rat HSCs were cultured in vitro and the effect of bilirubin treatment on their activation was assessed, including the effects on ROS production, $\alpha$-smooth 
muscle actin ( $\alpha$-SMA) and ECM remodeling-associated gene expression, cell proliferation and apoptosis. These results suggested that within the normal physiological concentration range, bilirubin may exert beneficial effects.

\section{Materials and methods}

Reagents and antibodies. Dulbecco's modified Eagle's medium (DMEM; HyClone; GE Healthcare Life Sciences, Logan, UT, USA) served as the cell culture medium. Fetal bovine serum (FBS) was purchased from Gibco; Thermo Fisher Scientific, Inc. (Waltham, MA, USA). Bilirubin was obtained from Sigma-Aldrich; Merck Millipore (Darmstadt, Germany). 2',7'-dichlorofluorescin diacetate (DCFH-DA) was obtained from eBioscience, Inc. (San Diego, CA, USA). A rabbit anti-rat $\alpha$-SMA primary antibody (cat. no. ab5694), a rabbit anti-GAPDH antibody (cat. no. ab9485) and a fluorescein isothiocyanate (FITC)-labeled goat anti-rat secondary antibody (cat. no. ab33110) were obtained from Abcam (Cambridge, UK). Angiotensin converting enzyme chromogenic reagents were obtained from Wuhan Boster Biological Technology, Ltd. (Wuhan, China). The Cell Counting Kit-8 (CCK-8) was purchased from Dojindo Molecular Technologies, Inc. (Kumamoto, Japan). The Apoptosis assay kit was obtained from Hangzhou MultiSciences (Lianke) Biotech Co., Ltd. (Hangzhou, China).

Cell culture. HSCs were isolated from 20 six-week-old Male Sprague-Dawley rats obtained from the Laboratory Animal Center at Wenzhou Medical College (Wenzhou, China). The care and use of animals in this study fully complied with relevant governmental and institutional requirements, guidelines and policies (15). Ethical approval was obtained from the Human Ethics Committee of Wenzhou Medical University (Wenzhou, China). The animals were sacrificed by intraperitoneal injection of $3 \%$ pentobarbital sodium $(60 \mathrm{mg} / \mathrm{kg}$ body weight, purchased from Beijing Propbs Biotechnology Co., Ltd.). The cell isolation method used was as previously described (16). Isolated HSCs were cultured in DMEM with L-glutamine and 10\% FBS. The medium was replaced every 2-3 days. Following primary culture for $\sim 7$ days, the cells underwent rapid proliferation. Experimental assays were conducted with fifth generation (passage) activated HSCs.

ROS assay. Cultured HSCs were adjusted to $1 \times 10^{5}$ cells $/ \mathrm{ml}$ and $100 \mu \mathrm{l} /$ well was seeded into 96-well plates. Bilirubin was diluted in DMEM with 10\% FBS and added at concentrations of $0,1,10$ or $20 \mathrm{mg} / \mathrm{l}$. After $24 \mathrm{~h}$ of treatment, the medium was aspirated. DCFH-DA diluted in DMEM at $10 \mu \mathrm{mol} / 1$ was added to each well and incubated for $30 \mathrm{~min}$. Cells were subsequently rinsed twice with DMEM and observed under a fluorescence microscope.

Western blot analysis. Isolated HSCs were treated with 0, 1,10 or $20 \mathrm{mg} / \mathrm{l}$ bilirubin for $24 \mathrm{~h}$, and subsequently lysed with cell lysis buffer (cat. no. P0013; Beyotime Institute of Biotechnology, Haimen, China) for $30 \mathrm{~min}$ on ice, and centrifuged at $5,000 \times \mathrm{g}$ for $15 \mathrm{~min}$ at $4^{\circ} \mathrm{C}$ to isolate the proteins in the supernatant. A total of $50 \mu \mathrm{g}$ isolated proteins were loaded onto $10 \%$ SDS-PAGE gels. Following SDS-PAGE,
Table I. Primer sequences for polymerase chain reaction detection of mRNA expression.

\begin{tabular}{lll}
\hline Gene & Direction & \multicolumn{1}{c}{ Sequence (5'-3') } \\
\hline TIMP-1 & Forward & CCTCTGGCATCCTCTTGTTG \\
& Reverse & CGCTGGTATAAGGTGGTCTC \\
MMP-2 & Forward & CCCCTATCTACACCTACACCAA \\
& Reverse & CACCACGGATCTGAGCAAT \\
GAPDH & Forward & GAGGACCAGGTTGTCTCCTG \\
& Reverse & GGATGGAATTGTGAGGGAGA \\
\hline
\end{tabular}

TIMP-1, tissue inhibitor of matrix metalloproteinase-1; MMP-2, matrix metalloproteinase-2.

the separated proteins were transferred to a polyvinylidene difluoride membrane. Membranes were subsequently blocked for $1 \mathrm{~h}$ with 5\% non-fat milk in TBS with Tween-20 (cat. no. K873-500ML, Shanghai Haoran Bio Technologies Co., Ltd., Shanghai, China). The anti-GAPDH antibody diluted at 1:200 and the anti- $\alpha$-SMA antibody diluted at 1:100 were added to the membrane and incubated overnight at $4^{\circ} \mathrm{C}$. Subsequently, the membrane was washed three times with TBS with Tween-20 for 10 min; the secondary antibody was added at a 1:30,000 dilution and incubated at $37^{\circ} \mathrm{C}$ for $1 \mathrm{~h}$. Following this, the membrane was washed again and treated with chromogenic reagents to visualize the proteins. The western blots were scanned and analyzed using the Gel-Pro Analyzer system (version, 4.5; Media Cybernetics, Inc. (Rockville, MD, USA).

Reverse transcription-polymerase chain reaction (RT-PCR) analysis of mRNA expression. HSCs were treated with various concentrations of bilirubin for $24 \mathrm{~h}$ and collected for RNA isolation (TRIzol kit, Invitrogen; Thermo Fisher Scientific, Inc., Waltham, MA, USA), cDNA synthesis (Beijing Dingguo Changsheng Biotechnology Co., Ltd., Beijing, China) and PCR detection (PCR kit cat. no. P3012; Guangdong Dongsheng Biotech Co., Ltd., Guangzhou, China) of mRNA expression of matrix metalloproteinase (MMP-2) and tissue inhibitor of matrix metalloproteinase-1 (TIMP-1). GAPDH served as an internal control. The primers used in PCR reactions are presented in Table I. PCR conditions were as follows: Initial denaturation at $94^{\circ} \mathrm{C}$ for $5 \mathrm{~min}$, followed by 40 cycles of denaturation for $30 \mathrm{sec}$ at $94^{\circ} \mathrm{C}$, annealing for $30 \mathrm{sec}$ at $52.1^{\circ} \mathrm{C}$ and extension for $45 \mathrm{sec}$ at $72^{\circ} \mathrm{C}$. The software used for densitometry was ImageJ (version, 1.48u; National Institutes of Health, Bethesda, MD, USA).

Cell proliferation assay. HSCs were seeded at $1 \times 10^{4}$ cells/well in 96-well plates and cultured in serum-free DMEM for $24 \mathrm{~h}$ prior to treatment with bilirubin. The final concentrations of bilirubin were $0,1,10$ and $20 \mathrm{mg} / 1$, with five replicates per concentration. After treatment for $24 \mathrm{~h}, 80 \mu \mathrm{l} \mathrm{CCK}-8$ solution was added to each well and incubated for $2 \mathrm{~h}$. The absorbance was read at a wavelength of $450 \mathrm{~nm}$ using a plate reader, and cell proliferation rates were calculated as previously described (16). 


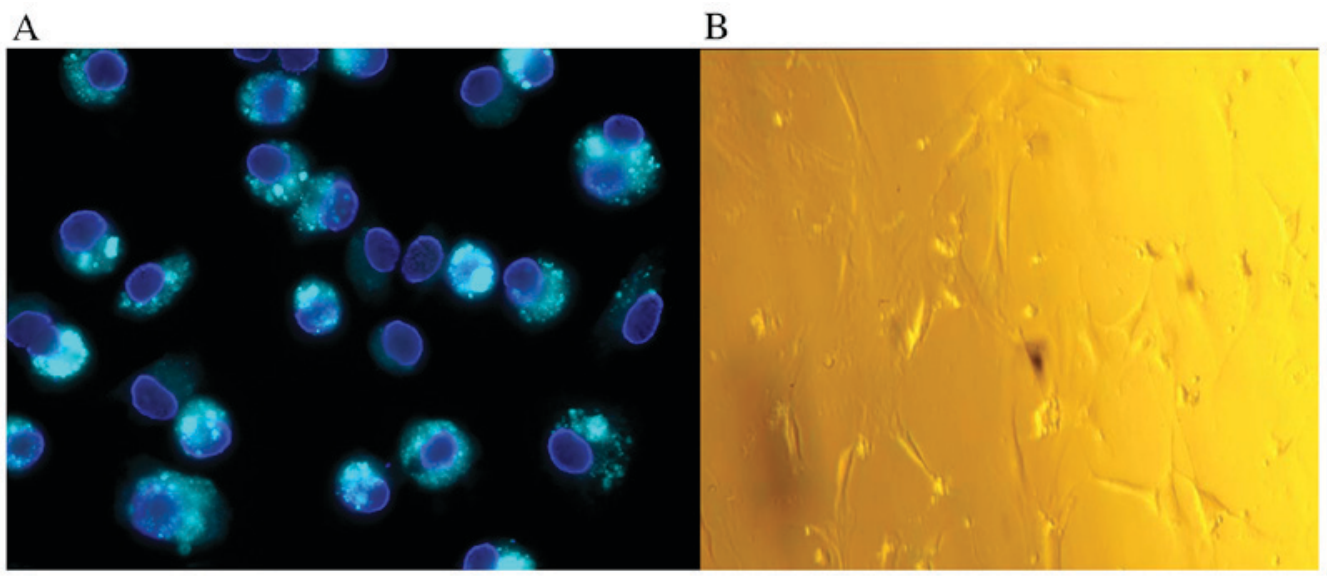

Figure 1. Morphology of HSCs in primary culture. (A) Representative image of a fluorescent rat primary HSC culture at day 2 (magnification, x600). (B) Representative image of a rat primary HSC culture at day 8 (magnification, x200). HSC, hepatic stellate cell.
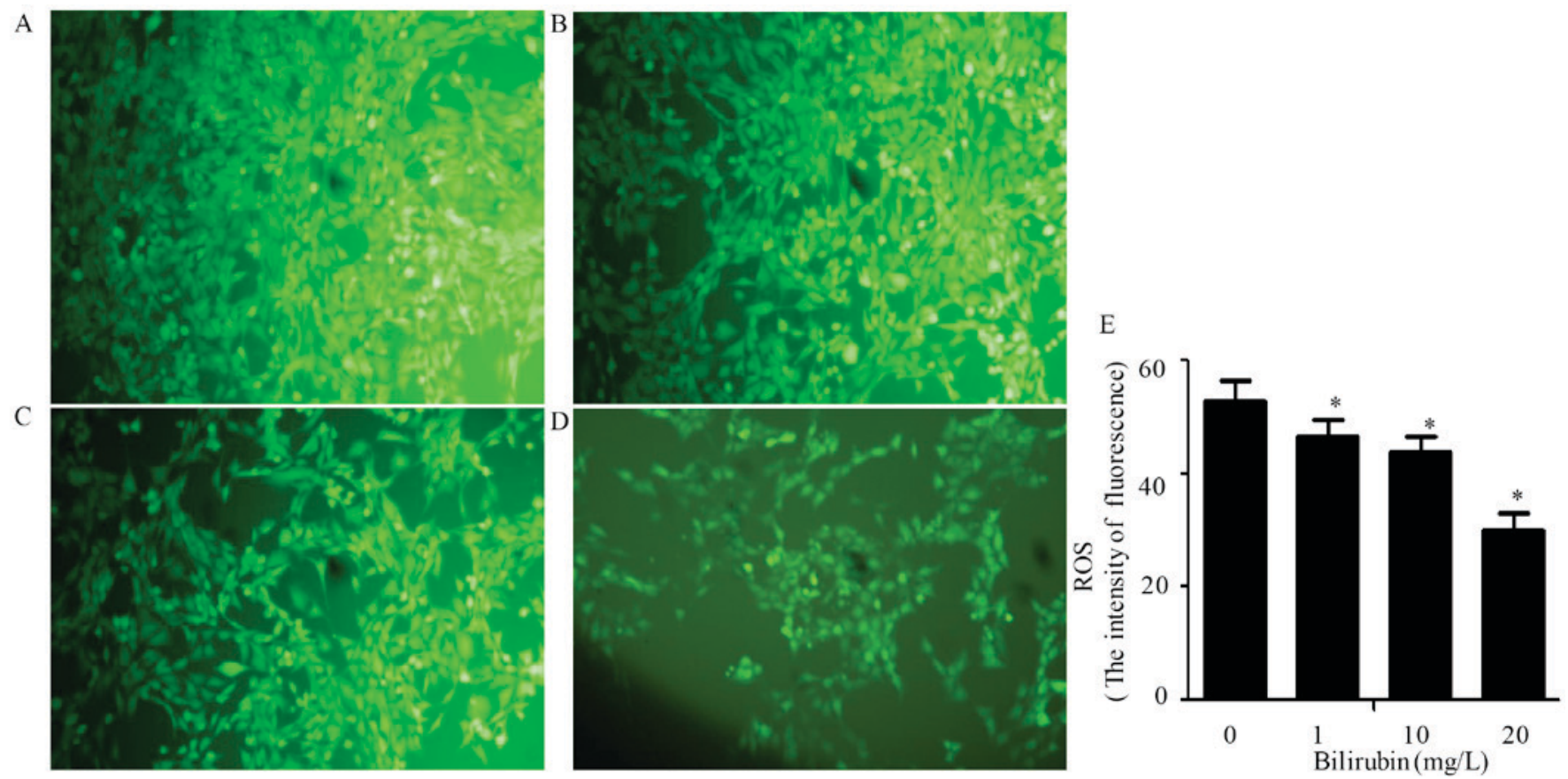

Figure 2. Bilirubin reduces reactive oxygen species production in hepatic stellate cells. Cells were cultured in medium containing bilirubin for $24 \mathrm{~h}$ prior to incubation for 30 min with $10 \mu \mathrm{M}$ 2',7'-dichlorofluorescin diacetate. Representative images of cells treated with (A) 0 (B) 1 (C) 10 and (D) $20 \mathrm{mg} / 1$ bilirubin. Magnification, $x 100$. (E) Quantification of the cellular fluorescence intensity. Data are presented as the mean \pm standard deviation $(\mathrm{n}=3)$. ${ }^{*} \mathrm{P}<0.05$ vs. control.

Apoptosis assay. Following bilirubin treatment, $22 \times 10^{5} \mathrm{HSCs}$ per sample were harvested by trypsinization, centrifuged at $1000 \times \mathrm{g}$ for $5 \mathrm{~min}$ at room temperature, washed twice with PBS and resuspended in $200 \mu \mathrm{l}$ binding buffer. Following this, $5 \mu \mathrm{l}$ Annexin V-FITC and $5 \mu \mathrm{l}$ propidium iodide were added to each sample. The samples were incubated at room temperature for $20 \mathrm{~min}$ in the dark prior to flow cytometric analysis, with an excitation wavelength of $488 \mathrm{~nm}$ and an emission wavelength of $530 \mathrm{~nm}$. The software used for flow cytometric analysis was CellQuest Pro (version, 5.1; BD Biosciences, Franklin Lakes, NJ, USA).

Statistical analysis. All data are expressed as the mean \pm standard deviation. Differences between groups were analyzed by one-way analysis of variance using SPSS software version
16.0 (SPSS, Inc., Chicago, IL, USA). No post hoc analysis was conducted. $\mathrm{P}<0.05$ was considered to indicate a statistically significant difference.

\section{Results}

Morphological alterations in HSCs during primary culture. HSCs, isolated with a yield of $1-2 \times 10^{7}$ cells/rat, demonstrated $>95 \%$ viability, as revealed by trypan blue staining. Freshly isolated HSCs were rounded in shape and contained multiple cytoplasmic lipid droplets. On excitation with light at a wavelength of $328 \mathrm{~nm}$, the droplets produced a green-blue fluorescence (Fig. 1A). Following primary culture for 2-3 days, cells became adherent. At days 5-7, cells appeared to lose their capability for lipid storage, acquired a star-like shape 

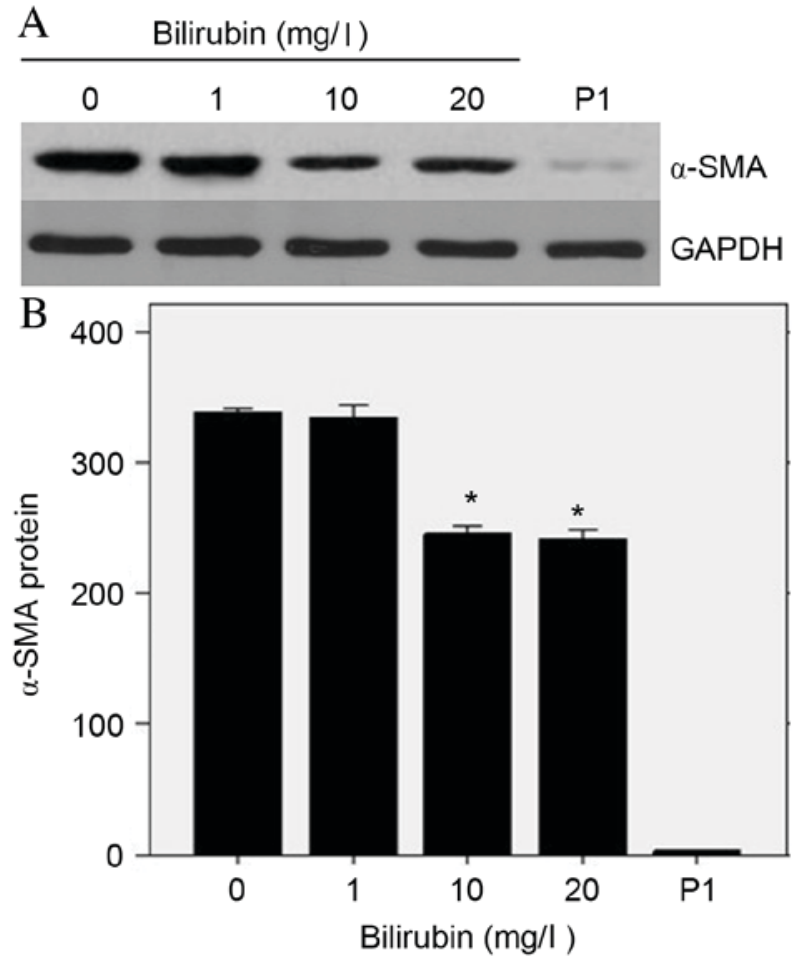

Figure 3. $\alpha$-SMA protein expression levels in HSCs treated with bilirubin. Isolated HSCs at passage 5 were cultured for $24 \mathrm{~h}$ with bilirubin prior to protein isolation. (A) Representative western blot image. GAPDH served as an internal control. (B) Quantification of western blotting. Data are presented as the mean \pm standard deviation $(\mathrm{n}=3)$. P1, isolated HSCs at passage 1 served as a control. $\alpha$-SMA, $\alpha$-smooth muscle actin; HSCs, hepatic stellate cells. " $\mathrm{P}<0.05$ vs. control.

and demonstrated rapid proliferation (Fig. 1B). Gradually, the isolated quiescent HSCs in the primary culture transitioned to activated HSCs. These activated HSCs appeared to exhibit features of an effective model for the study of HSC-associated liver diseases. These cells transformed into myofibroblast-like cells with active contractility, and appeared to induce fibrogenesis, matrix degradation and proliferation. Activated HSC cultures at passage 5 were used for all subsequent experiments unless otherwise stated.

Effect of bilirubin on ROS production. ROS production is an important feature of HSC activation, and generated ROS may in turn stimulate the activation of HSCs. Bilirubin was added to the HSC culture medium at concentrations of 0,1 , 10 or $20 \mathrm{mg} / \mathrm{l}$ for $24 \mathrm{~h}$. Following this, DCFH-DA was used to assess ROS production. As bilirubin concentration increased, the cellular fluorescence intensity decreased (Fig. 2). The ROS production was measured using an arbitrary fluorescence unit, and the values were $53.6 \pm 6.3,46.7 \pm 5.6,44.7 \pm 5.5$ and $31.2 \pm 6.1$, respectively $(\mathrm{F}=187.527, \mathrm{P}=0.036)$. These findings indicated that bilirubin reduced ROS production in HSCs in a dose-dependent manner.

Effect of bilirubin on $\alpha$-SMA protein expression levels. The expression levels of $\alpha$-SMA in HSCs reflect their transition to myofibroblast-like cells and is therefore a marker of HSC activation. Western blot analysis demonstrated a dramatic decrease
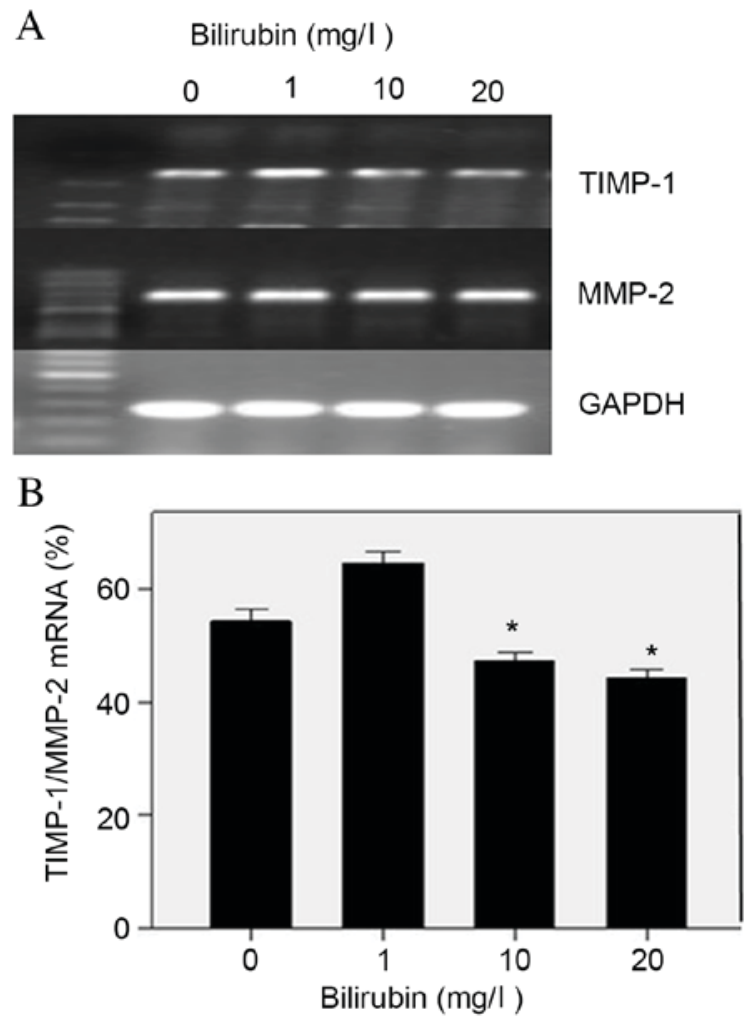

Figure 4. TIMP-1 and MMP-2 mRNA expression levels in HSCs treated with bilirubin. Isolated HSCs at passage 5 were cultured with bilirubin for $24 \mathrm{~h}$ prior to RNA isolation. (A) Representative gel revealing TIMP-1 and MMP-2 mRNA expression levels following treatment, with GAPDH serving as the internal control. (B) Densitometry of the polymerase chain reaction gel revealed the mRNA ratios of TIMP-1 and MMP-2. Data are presented as the mean \pm standard deviation $(\mathrm{n}=3)$. TIMP-1, tissue inhibitor of matrix metalloproteinase-1; MMP-2, matrix metalloproteinase-2; HSCs, hepatic stellate cells. ${ }^{*} \mathrm{P}<0.05$ vs. control.

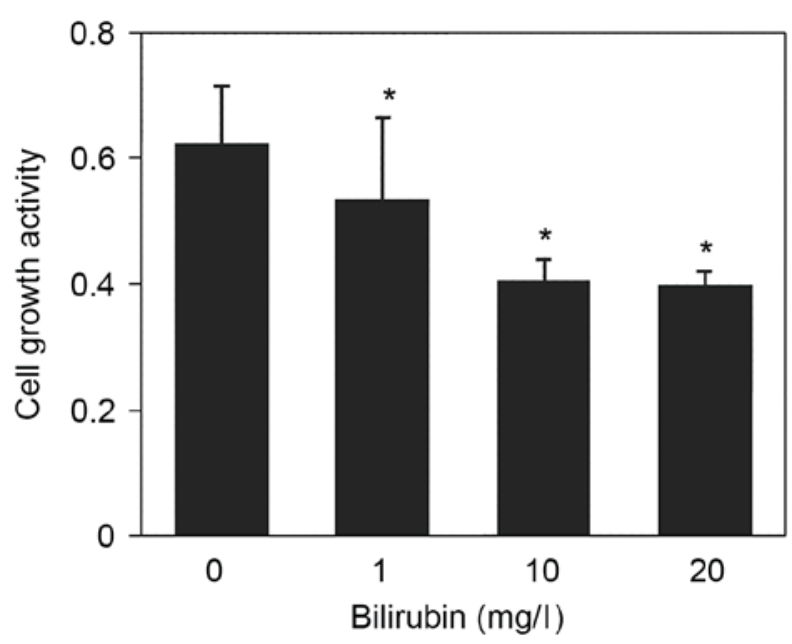

Figure 5. Bilirubin inhibits proliferation of hepatic stellate cells. Cells were seeded at an equal density and cultured for $24 \mathrm{~h}$ with bilirubin prior to a Cell Counting Kit- 8 assay. Cell growth activity decreased following bilirubin treatment in a dose-dependent manner. Data are presented as the mean \pm standard deviation $(\mathrm{n}=5) .{ }^{*} \mathrm{P}<0.05$ vs. control.

in $\alpha$-SMA protein expression levels as bilirubin concentration increased (Fig. 3A). At bilirubin concentrations of $0,1,10$ and $20 \mathrm{mg} / \mathrm{l}$, and passage $1 \mathrm{HSCs}$, the relative $\alpha$-SMA protein 
A

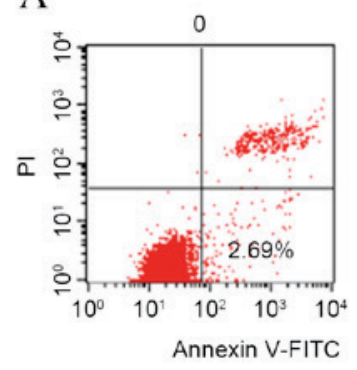

Bilirubin (mg/l)
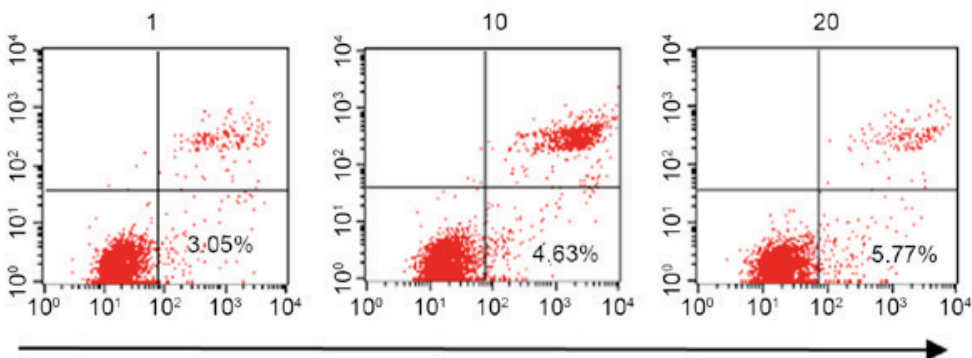

B

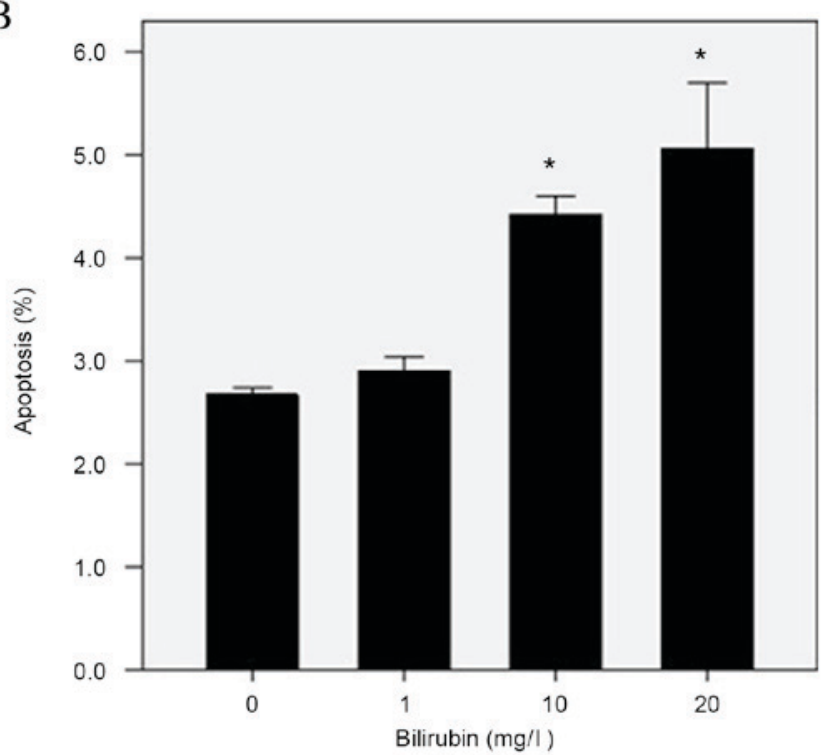

Figure 6. Bilirubin promotes apoptosis of hepatic stellate cells. Cells were cultured in medium with bilirubin for $24 \mathrm{~h}$ and subsequently stained with annexin V-FITC and PI, followed by flow cytometric analysis. (A) Apoptotic cells are indicated as a percentage of gated cells. A representative experiment is presented. (B) Analysis of apoptosis assay results. Data are presented as the mean \pm standard deviation $(\mathrm{n}=3)$. FITC, fluorescein isothiocyanate; PI, propidium iodide. ${ }^{*} \mathrm{P}<0.05$ vs. control.

expression levels were $339 \pm 2,336 \pm 10,246 \pm 7,242 \pm 5$ and $3.7 \pm 0.3$ respectively (Fig. 3B). A one-way ANOVA determined the $\mathrm{F}$ ratio of these groups to be $191.107(\mathrm{P}=0.042)$, thus indicating significantly reduced expression levels of $\alpha$-SMA in activated HSCs treated with bilirubin.

Effect of bilirubin on ECM remodeling-associated gene expression. Upon activation, HSCs express a combination of MMPs and TIMPs to remodel the ECM and facilitate the progression of liver fibrosis. With increased concentrations of bilirubin in HSC culture, the mRNA expression levels of MMP-2 remained relatively unaltered, as indicated by RT-PCR (Fig. 4A); however, the mRNA ratio of TIMP-1/MMP-2 decreased significantly, from 54 to 44\% ( $\mathrm{F}=73.4$; $\mathrm{P}=0.047$; Fig. 4B). TIMP-1 may inhibit the degradation of fibrillary collagen, therefore these findings indicated that bilirubin may promote ECM degradation during HSC activation.

Effect of bilirubin on HSC proliferation. Cell proliferation is an important component of HSC activation that increases the number of active cells. The effect of bilirubin on HSC proliferation was examined by $\mathrm{CCK}-8$ assay. Following treatment with $0,1,10$ and $20 \mathrm{mg} / 1$ bilirubin, HSC proliferation activity was $0.624 \pm 0.092,0.536 \pm 0.127,0.407 \pm 0.033$ and $0.399 \pm 0.022$, respectively ( $\mathrm{F}=13.454 ; \mathrm{P}=0.041 ;$ Fig. 5$)$. These results indicated that increased bilirubin appeared to have an inhibitory effect on HSC proliferation.

Effect of bilirubin on HSC apoptosis. Apoptosis of HSCs may account for the decrease in activated HSCs during the resolution phase of hepatic fibrosis. As bilirubin concentrations increased from 0 to $20 \mathrm{mg} / \mathrm{l}$, the percentage of apoptotic HSCs increased from 2.69 to $5.77 \%$ (Fig. 6A). Repeated assays demonstrated that bilirubin treatment resulted in increased apoptosis of HSCs in a dose-dependent manner ( $\mathrm{F}=34.731 ; \mathrm{P}=0.033$; Fig. 6B).

\section{Discussion}

To the best of our knowledge, this is the first study to demonstrate that bilirubin treatment at physiological concentrations attenuates the activation of rat HSCs in vitro. Bilirubin treatment exerted numerous effects on HSCs, including reduced ROS production, decreased protein expression levels of the HSC activation marker $\alpha$-SMA and the ECM remodeling gene TIMP-1, inhibited proliferation and increased apoptosis. These characteristics indicated inhibition of HSC activation.

HSC activation is a pleiotropic and tightly regulated response that occurs in a reproducible sequence. It may be 
divided into two phases: Initiation and perpetuation (3). The initiation phase is associated with rapid alterations in gene expression in response to stimulations from liver injury, including increased expression levels of the contractile protein $\alpha$-SMA, and upregulation of MMP- 2 and TIMP-1 to remodel the ECM. The perpetuation phase is a dynamic process, which consists of cellular events that amplify the activated phenotype, including HSC proliferation. Apoptosis of HSCs is typical during fibrosis resolution, which facilitates clearance of the activated HSCs (17). The results of the present study revealed that bilirubin treatment altered the gene expression and growth rate of HSCs, thus impacting the initiation and perpetuation phases of HSC activation. Furthermore, bilirubin treatment promoted HSC apoptosis, and may influence the resolution of liver fibrosis.

The underlying molecular mechanisms of bilirubin-mediated downregulation of HSC activation involve its antioxidant activity. The primary source of ROS in activated HSCs is the plasma membrane-associated nicotinamide adenine dinucleotide phosphate (NADPH) oxidase (18). HSC cultures from NADPH oxidase-deficient mice were demonstrated to exhibit reduced ROS production, and following bile duct ligation, these mice were protected from liver injury and underwent fibrosis to a lesser degree (12). Additionally, bilirubin has been revealed to be a potent inhibitor of NADPH oxidase $(19,20)$. Thus, the inhibition of HSC activation by bilirubin in the present study may have been mediated via inhibition of NADPH oxidase activity in HSCs.

Bilirubin is the breakdown product of heme, present in RBCs. The degradation of heme in humans is catalyzed by the enzyme heme oxygenase (HO), which is the rate-limiting step in the formation of bilirubin (21). Consistent with the present findings, induction of $\mathrm{HO}$ expression in human hepatic myofibroblasts had an antifibrogenic effect, including inhibition of proliferation and procollagen expression, which was ascribed to the increased levels of bilirubin (22). Additionally, these results indicate that bilirubin may attenuate HSC activation in rats and humans. Furthermore, adeno-associated virus-mediated HO-1 gene transfer was revealed to suppress the progression of micronodular cirrhosis in rats (23), suggesting that these in vitro data may be applicable in vivo, and that bilirubin may be beneficial for the treatment of hepatic fibrosis.

Lanone et al (24) demonstrated the potent antioxidant effects of bilirubin, which appeared to protect against endotoxic shock in rats by inhibiting NADPH oxidase. However, their study was conducted in genetically modified jaundiced rats, which limited its clinical application and significance. Visible jaundice typically occurs when total bilirubin concentration is $>25 \mathrm{mg} / \mathrm{l}$. The results of the present study were generated in assays using bilirubin at physiological concentrations, which demonstrated its dose-dependent effect. Therefore, although cytotoxic at high concentrations, bilirubin may potentially contribute to antifibrotic therapies within its physiological levels.

In conclusion, a previous study have suggested that specific homologs of bilirubin, combined with additional therapeutic agents, may be beneficial for the clinical treatment of hepatic fibrosis (19). The present study demonstrated that low concentrations of bilirubin may inhibit the activation of isolated HSCs, including ROS production, fibroblast transition, ECM remodeling, cell proliferation and apoptosis. Further in vivo studies investigating the effect of bilirubin on HSC activation and hepatic fibrosis may reveal potential novel strategies for the use of bilirubin in antifibrogenic therapy.

\section{Acknowledgements}

The present study was supported in part by the Zhejiang Province Key Surgery Projects (Zhejiang High-Tech; grant. no. 2008-255) and Zhejiang Provincial Natural Science Foundation (grant no. LY16H030014).

\section{References}

1. Geerts A: History, heterogeneity, developmental biology, and functions of quiescent hepatic stellate cells. Semin Liver Dis 21: 311-335, 2001.

2. Brandão DF, Ramalho LN, Ramalho FS, Zucoloto S, Martinelli Ade L and Silva Ode C: Liver cirrhosis and hepatic stellate cells. Acta Cir Bras 21 (Suppl 1): S54-S57, 2006.

3. Li JT, Liao ZX, Ping J, Xu D and Wang H: Molecular mechanism of hepatic stellate cell activation and antifibrotic therapeutic strategies. J Gastroenterol 43: 419-428, 2008.

4. Atzori L, Poli G and Perra A: Hepatic stellate cell: A star cell in the liver. Int J Biochem Cell Biol 41: 1639-1642, 2009.

5. Tomaro ML and Batlle AM: Bilirubin: Its role in cytoprotection against oxidative stress. Int J Biochem Cell Biol 34: 216-220, 2002.

6. Washington K, Wright K, Shyr Y, Hunter EB, Olson S and Raiford DS: Hepatic stellate cell activation in nonalcoholic steatohepatitis and fatty liver. Hum Pathol 31: 822-828, 2000.

7. Stocker R, Yamamoto Y, McDonagh AF, Glazer AN and Ames BN: Bilirubin is an antioxidant of possible physiological importance. Science 235: 1043-1046, 1987.

8. Wu TW, Fung KP, Wu J, Yang CC and Weisel RD: Antioxidation of human low density lipoprotein by unconjugated and conjugated bilirubins. Biochem Pharmacol 51: 859-862, 1996.

9. Yamaguchi T, Horio F, Hashizume T, Tanaka M, Ikeda S, Kakinuma A and Nakajima $\mathrm{H}$ : Bilirubin is oxidized in rats treated with endotoxin and acts as a physiological antioxidant synergistically with ascorbic acid in vivo. Biochem Biophys Res Commun 214: 11-19, 1995.

10. Hansen TW: Bilirubin production, breast-feeding and neonatal jaundice. Acta Paediatr 90: 716-717, 2001.

11. Gitto E, Reiter RJ, Karbownik M, Tan DX, Gitto P, Barberi S and Barberi I: Causes of oxidative stress in the pre- and perinatal period. Biol Neonate 81: 146-157, 2002.

12. Bataller R, Schwabe RF, Choi YH, Yang L, Paik YH, Lindquist J, Qian T, Schoonhoven R, Hagedorn CH, Lemasters JJ and Brenner DA: NADPH oxidase signal transduces angiotensin II in hepatic stellate cells and is critical in hepatic fibrosis. J Clin Invest 112: 1383-1394, 2003.

13. Zhong Z, Froh M, Wheeler MD, Smutney O, Lehmann TG and Thurman RG: Viral gene delivery of superoxide dismutase attenuates experimental cholestasis-induced liver fibrosis in the rat. Gene Ther 9: 183-191, 2002.

14. Ahmad A and AhmadR: Resveratrolmitigate structuralchanges and hepatic stellate cell activation in N'-nitrosodimethylamine-induced liver fibrosis via restraining oxidative damage. Chem Biol Interact 221: 1-12, 2014.

15. Yu FX, Teng YY, Zhu QD, Zhang QY and Tang YH: Inhibitory effects of capsaicin on hepatic stellate cells and liver fibrosis. Biochem Cell Biol 92: 406-412, 2014.

16. Yu F, Su L, Ji S, Zhang S, Yu P, Zheng Y and Zhang Q: Inhibition of hepatic stellate cell activation and liver fibrosis by fat-specific protein 27. Mol Cell Biochem 369: 35-43, 2012.

17. Elsharkawy AM, Oakley F and Mann DA: The role and regulation of hepatic stellate cell apoptosis in reversal of liver fibrosis. Apoptosis 10: 927-939, 2005.

18. Choi SS, Sicklick JK, Ma Q, Yang L, Huang J, Qi Y, Chen W, Li YX, Goldschmidt-Clermont PJ and Diehl AM: Sustained activation of Rac1 in hepatic stellate cells promotes liver injury and fibrosis in mice. Hepatology 44: 1267-1277, 2006.

19. McCarty MF, Barroso-Aranda J and Contreras F: Genistein and phycocyanobilin may prevent hepatic fibrosis by suppressing proliferation and activation of hepatic stellate cells. Med Hypotheses 72: 330-332, 2009. 
20. Jiang F, Roberts SJ, Datla Sr and Dusting GJ: NO modulates NADPH oxidase function via heme oxygenase-1 in human endothelial cells. Hypertension 48: 950-957, 2006.

21. Kikuchi G, Yoshida T and Noguchi M: Heme oxygenase and heme degradation. Biochem Biophys Res Commun 338: 558-567, 2005.

22. Li L, Grenard P, Nhieu JT, Julien B, Mallat A, Habib A and Lotersztajn S: Heme oxygenase-1 is an antifibrogenic protein in human hepatic myofibroblasts. Gastroenterology 125: 460-469, 2003.
23. Tsui TY, Lau CK, Ma J, Glockzin G, Obed A, Schlitt HJ and Fan ST: Adeno-associated virus-mediated heme oxygenase-1 gene transfer suppresses the progression of micronodular cirrhosis in rats. World J Gastroenterol 12: 2016-2023, 2006.

24. Lanone S, Bloc S, Foresti R, Almolki A, Taillé C, Callebert J, Conti M, Goven D, Aubier M, Dureuil B, et al: Bilirubin decreases nos 2 expression via inhibition of NAD(P)H oxidase: Implications for protection against endotoxic shock in rats. FASEB J 19: 1890-1892, 2005. 